\author{
MITSUBISHI ELECTRIC RESEARCH LABORATORIES \\ http://www.merl.com
}

\title{
A multidirectional capacitive proximity sensor array
}

Long, J.; Wang, B.

TR2014-031 June 2014

\begin{abstract}
This paper presents a multi-directional capacitive proximity sensor array based on band-stop filter. The band- stop filter is composed of four decoupled LC resonant circuits, so each stop band is determined by one capacitive sensor and its corresponding lumped inductor, respectively. Therefore, the change in capacitance can be obtained by measuring the change of the notches of the band-stop response. The filter is fabricated into a cubic shape for sensing different directions. Experiment results show that the fabricated sensor can detect objects coming from all four directions simultaneously by measuring the transmission at frequencies near four notches. (2014) COPYRIGHT Society of Photo-Optical Instrumentation Engineers (SPIE). Downloading of the abstract is permitted for personal use only.
\end{abstract}

SPIE Proceedings Vol 9116

This work may not be copied or reproduced in whole or in part for any commercial purpose. Permission to copy in whole or in part without payment of fee is granted for nonprofit educational and research purposes provided that all such whole or partial copies include the following: a notice that such copying is by permission of Mitsubishi Electric Research Laboratories, Inc.; an acknowledgment of the authors and individual contributions to the work; and all applicable portions of the copyright notice. Copying, reproduction, or republishing for any other purpose shall require a license with payment of fee to Mitsubishi Electric Research Laboratories, Inc. All rights reserved.

Copyright (C) Mitsubishi Electric Research Laboratories, Inc., 2014

201 Broadway, Cambridge, Massachusetts 02139 



\title{
A multidirectional capacitive proximity sensor array
}

\author{
Jiang Long ${ }^{a, b}$ and Bingnan Wang ${ }^{a, *}$ \\ ${ }^{a}$ Mitsubishi Electric Research Laboratories, 201 Broadway, Cambridge, MA 02139 \\ ${ }^{b}$ University of California, San Diego, 9500 Gilman Dr, La Jolla, CA, 92093
}

\begin{abstract}
This paper presents a multi-directional capacitive proximity sensor array based on band-stop filter. The bandstop filter is composed of four decoupled LC resonant circuits, so each stop band is determined by one capacitive sensor and its corresponding lumped inductor, respectively. Therefore, the change in capacitance can be obtained by measuring the change of the notches of the band-stop response. The filter is fabricated into a cubic shape for sensing different directions. Experiment results show that the fabricated sensor can detect objects coming from all four directions simultaneously by measuring the transmission at frequencies near four notches.
\end{abstract}

Keywords: proximity sensor, capacitive sensor, multi-directional sensor

\section{INTRODUCTION}

Capacitive sensor array has been applied to many areas, such as fingerprint sensing, robotic textile sensing, touch screen sensing, proximity sensing, imaging, medical care sensing, and security scanning . ${ }^{1-10}$ In general, the signal of a capacitive sensor array is processed with time division multiplexing ${ }^{1-9}$ However, the readout speed decreases with the increasing number of sensing element. Frequency domain multiplexing technique provides another way of increasing the readout efficiency ${ }^{11-18}$ An object can be sensed from the reflection of a transmission line,${ }^{11,12}$ but the information of all the elements are coupled together, resulting in a large uncertainty.

It is known that the resonance frequency can be used for measuring capacitance. It is possible to apply multi-resonance into capacitive sensor array signal processing, where each resonance corresponds to one sensing element, respectively. Previously such multi-resonance method has been demonstrated with split-ring resonator (SRR). ${ }^{17,18}$ With the help of SRR, the localized electric field is enhanced in the gap of the SRR so as the sensitivity is increased. On the other hand, the sensing area is limited to the small gap region of the SRR and the object has to be in contact with the sensor.

Here we present a multi-directional capacitive proximity sensor array based on band-stop filter design. A four-element capacitive proximity sensor array is integrated into a quad-band band-stop filter. Each stop band is determined by one capacitive sensor respectively. The capacitance can be obtained by measuring the change in transmission around the four notches. Such multi-direction capacitive proximity sensor is designed and fabricated. The measurement results shows the capability of detecting objects coming from four directions.

\section{THEORY AND DESIGN}

In general, the capacitive sensor is based on the change of the capacitive coupling between target object and sensor. The target changes surface charge distribution of the capacitive sensor, adding extra electric line between electrodes, thus increasing the capacitance. The closer the target, the larger the capacitance increase. A resonance can be obtained if it is connected with a series inductance. Then, the resonant frequency is dependent on the capacitance value.

One the other hand, microwave filter utilizes tuning capacitance for achieving different responses. ${ }^{19-21}$ In particular, band-stop filter exploits the series parallel-resonance tank and shunt series-resonance tank to form a band-stop response. It should be noticed that the shunt series-resonance can be replaced with the capacitive sensor in series with a inductor. This way, the capacitive sensor can be integrated into a band-stop filter. As shown in Fig. 1, a four-element sensor array are cascaded. Each capacitive sensing element is in series with

*E-mail: bwang@merl.com 


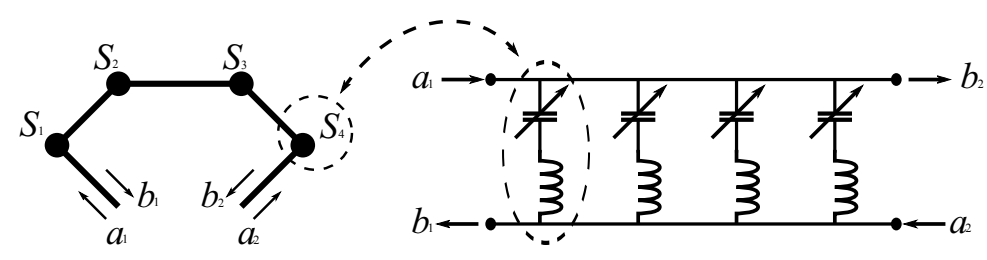

(a)

(b)

Figure 1. Integrating a capacitive sensor array into a band-stop filter. (a) is a series four-element capacitive sensor array. Each node $\left(S_{1}\right.$ to $\left.S_{4}\right)$ represents a sensor element, which is in series with a inductor to form resonance; (b) is the equivalent circuit. Each sensor element corresponds to one series LC tank.

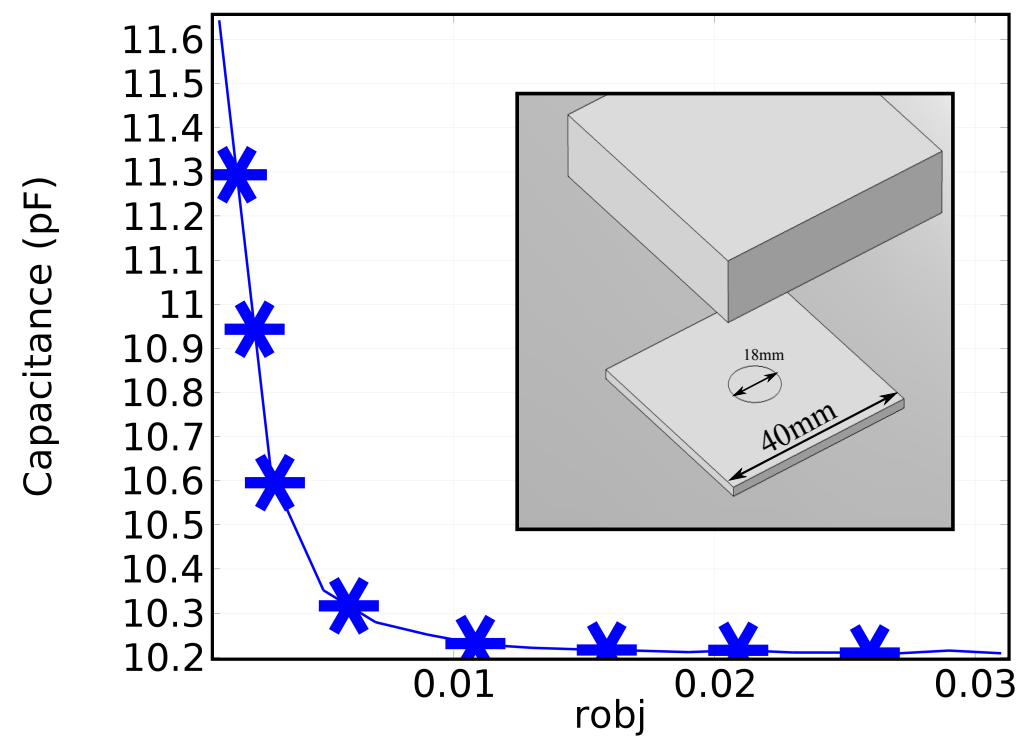

Figure 2. Simulation of the patch sensor and its capacitance change with the distance of an object.

an inductor, forming a series resonance. Thus the overall equivalent circuit can be given by the one shown in Fig. 1(b). From there we can see that the capacitance value is related to the central frequency of the stop band. Thus measurement of capacitance can be replaced by the measurement of other quantities, such as the stop band frequency, and transmission coefficient measurement at these frequencies. This enables frequency domain sensing method. Another advantage is that each resonant frequency of a sensing element can be controlled by the series inductance, while he the variation of capacitance is reflected by the perturbation of resonant frequency. This way the operating frequencies can be easily tuned.

Based on this principle, a four-element proximity sensor array working at different directions is designed. The capacitive structure is realized by a circular patch. The capacitance of the structure can be simulated with COMSOL Multiphysics. With an approaching target, the change in capacitance can also be modeled. Fig. 2 shows the numerical model and simulated capacitance change with the distance of an approaching object, which is a block of copper. The patch has a radius of $9 \mathrm{~mm}$. It is on the top of a $40 \mathrm{~mm}$ by $40 \mathrm{~mm}$ square, $1.2 \mathrm{~mm}$-thick FR4 substrate (relative permittivity $\epsilon=4.5$ ). The other side of the substrate is covered by copper as ground plane. A coax connector is placed as the center of the patch for excitation. The input impedance from the coax port is simulated. Distance of object to sensor is varied from $1 \mathrm{~mm}$ to $30 \mathrm{~mm}$. From the curve shown in Fig. 2, we can see that the capacitance of the sensor changes from $10.2 \mathrm{pF}$ to $11.6 \mathrm{pF}$.

Such capacitors can be integrated into a band-stop filter. As shown in Fig. 3, four capacitors with the same nominal value of $10.2 \mathrm{pF}$ are integrated in the filter. Each capacitor is connected to a transmission line through an inductor with different values. In order to relate each capacitor to a different resonance, the four stop band 


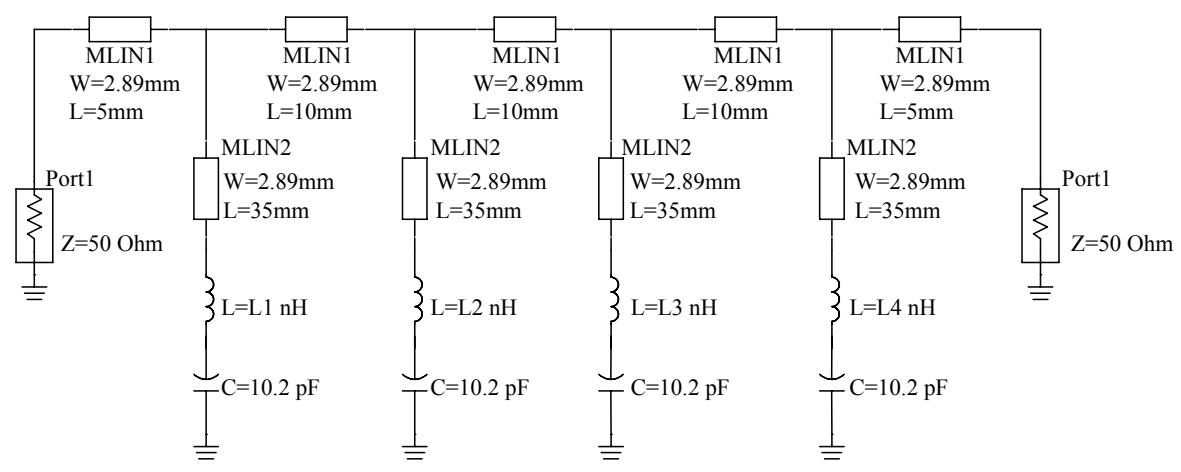

Figure 3. The schematic of sensor integrated band-stop filer circuit simulation. $C_{1}=10.2 \mathrm{pF}, L_{i}(i=1,2,3,4)$ is determined by different resonant frequencies.

should be separated far enough for good isolation. In the design, the stop bands are controlled by manipulating the product of inductance $(L)$ and capacitance $(C)$ as it directly determines the stop band frequency. In our design, the inductance values are chosen to be $82 \mathrm{nH}, 56 \mathrm{nH}, 27 \mathrm{nH}$, and $8.2 \mathrm{nH}$, respectively. The corresponding resonant frequencies are designed to be at $174 \mathrm{MHz}, 211 \mathrm{MHz}, 303 \mathrm{MHz}$, and $550 \mathrm{MHz}$, respectively. In order to simplify the design, all sensing elements are with the same dimension as simulated in Fig. 2, and all the capacitors and inductors are ideal components with infinite $\mathrm{Q}$ in the simulation. Besides the aforementioned capacitors and inductors, transmission line effect has been taken into account in the circuit simulation. There are two types of transmission lines in the simulation: MLIN1 and MLIN2. MLIN1 is for the feeding network, and MLIN2 is for connecting each of the $L C$ tank. They are all $50 \Omega$ transmission lines. In the simulation, four capacitors are swept from $10.2 \mathrm{pF}$ to $11.6 \mathrm{pF}$, respectively, representing the simulated capacitance change change as shown in Fig. 2.

The circuit simulation result is shown in Fig. 4. From the figures we can see that the four stop bands are decoupled in frequency spectrum, which shows the independent control of the resonant frequency. Also, with the variation of the capacitance in one element, the corresponding notch frequency changes, with all other notches practically unaffected. It should be noted that the simulated resonant frequencies deviate from previous calculations. This is because of the transmission line effect, especially the MLIN2 in Fig. 3. However, despite of the shifting of the resonant frequencies, it does not affect the isolation of the resonant frequencies. Therefore, it is still clear to see that all the capacitive sensing elements are decoupled with each other, and each of them is represented by one notch frequency.

\section{FABRICATION AND MEASUREMENT}

The sensor element and the feeding network are designed with Agilent Advanced Design System (ADS), which are shown in Fig. 5(a) and (b). The sensing element is designed on a 3-layer PCB, with the substrate of FR4. The top layer is the circular patch, which is connected to a $50 \Omega$ transmission line on the bottom layer through a via. The middle layer is the ground plane, which is $1.2 \mathrm{~mm}$ below the top layer and $0.3 \mathrm{~mm}$ above the bottom layer. The feeding network is a folded transmission line designed on a $1.6 \mathrm{~mm}$-thick 2-layer PCB (FR4 substrate). A folded shape is adopted because the sensors are arranged in different directions. All the dimensions are also listed in the figure. Four capacitive sensor elements are wrapped on a cube. The feeding network is connected to the four sensing elements from the inside of the cube. All the ground planes are soldered together. Two SMA connectors are mounted on the feeding network PCB. Fig. 5(c) and (d) are pictures of the fabricated four-element multi-directional capacitive proximity sensing array.

Measurements are done with a network analyzer. The setup is shown in Fig. 6. A target object (metal block) is placed in front of each face of the sensor. The S-parameters are recorded with different object-to-sensor distances from four different directions. In the measurements, S-parameters are recorded for the object with a distance ranging from $2 \mathrm{~mm}$ to $20 \mathrm{~mm}$ with a step size of $2 \mathrm{~mm}$. Fig. 6 shows the measured magnitude of $S_{21}$ for the object coming from different directions. By comparing the four subfigures, it is clear to see that object 


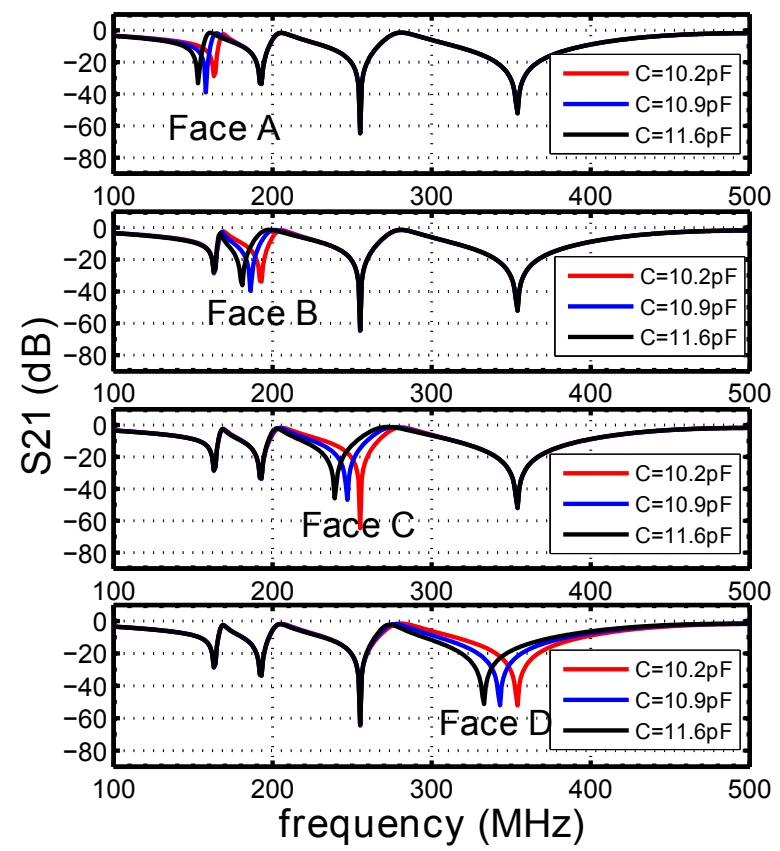

(a)

Figure 4. Transmission spectrum given by circuit simulations. The four sub-figures indicate the change in capacitance at four sensing elements with different inductance values: face $\mathrm{A}\left(L_{1}=82 \mathrm{nH}\right), \mathrm{B}\left(L_{2}=56 \mathrm{nH}\right), \mathrm{C}\left(L_{3}=27 \mathrm{nH}\right), \mathrm{D}$ $\left(L_{4}=8.2 \mathrm{nH}\right)$. In each subfigure, curves with different colors represent different capacitance values.

coming from different directions causes resonant frequency shift of the corresponding sensing element while keeps all other resonances unaffected. Compared with the simulation results shown in Fig. 4, the resonant frequency is different, which is due to the non-ideal capacitance and inductance, as well as the manual soldering during fabrication which causes extra parasitics.

Also the resonant frequency shifts to the lower band when the object approaches, which is due to the increase of capacitance. At a frequency slightly above the resonance, the magnitude of $S_{21}$ increases due to that shift in resonant frequency. Therefore, it is possible to detect the object by examining the magnitude of $S_{21}$ at those frequencies. Also, the most sensitive part is the frequency region closed to the resonance. Therefore, four frequencies which are right above each resonant frequency will give the best performance for sensing purpose. Base on measurement results in Fig. 7, $140 \mathrm{MHz}, 180 \mathrm{MHz}, 240 \mathrm{MHz}$ and $340 \mathrm{MHz}$ are chosen for $S_{21}$ magnitude sensing analysis. The change of $S_{21}$ at these four frequencies versus the distance of the target object is plotted for those cases where the object is approaching from different directions. In all the curves at a particular frequency, only one curve is obviously different from the other three, which indicate this frequency is sensitive only to objects in that direction. Also, from Fig. 8, the sensing range can be estimated. If the power measurement uncertainty is as low as $0.5 \mathrm{~dB}$, the sensing range of the designed sensor is about $8 \mathrm{~mm}$.

From the comparison between the measurement (Fig. 7) and the simulation (Fig. 4), we find that the quality factor of the resonance is significantly reduced. This is due to the dielectric loss in the substrate and ohmic loss in the metallic structures. This significantly affects the sensitivity. Different sensing structures with higher quality factor can be designed in order to improve the sensitivity. Besides, low loss substrate will also improve the sensitivity. 


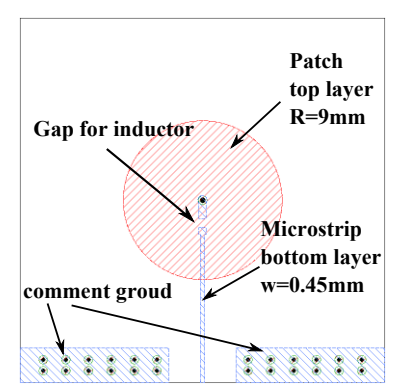

(a)

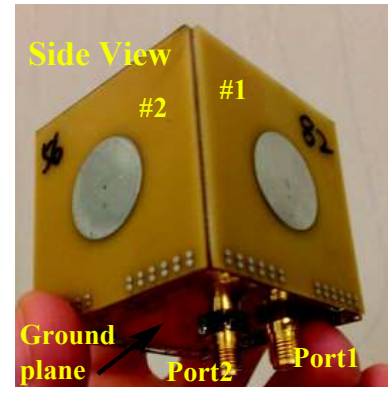

(c)

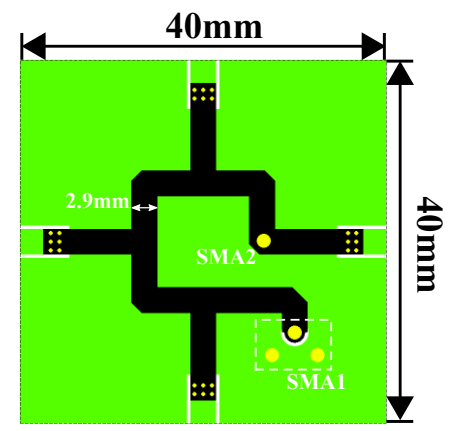

(b)

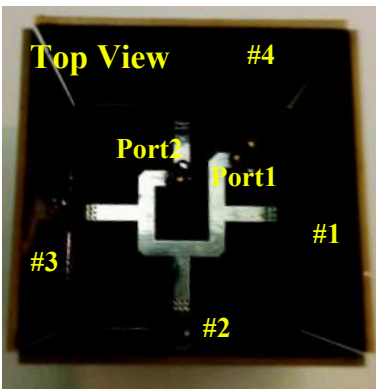

(d)

Figure 5. The designed layout and the fabricated sensor. (a)The layout of the sensor element; (b) the layout of the feeding network, with the top layer shown in black and bottom layer shown in green; (c)and (d) are pictures of the fabricated sensor.

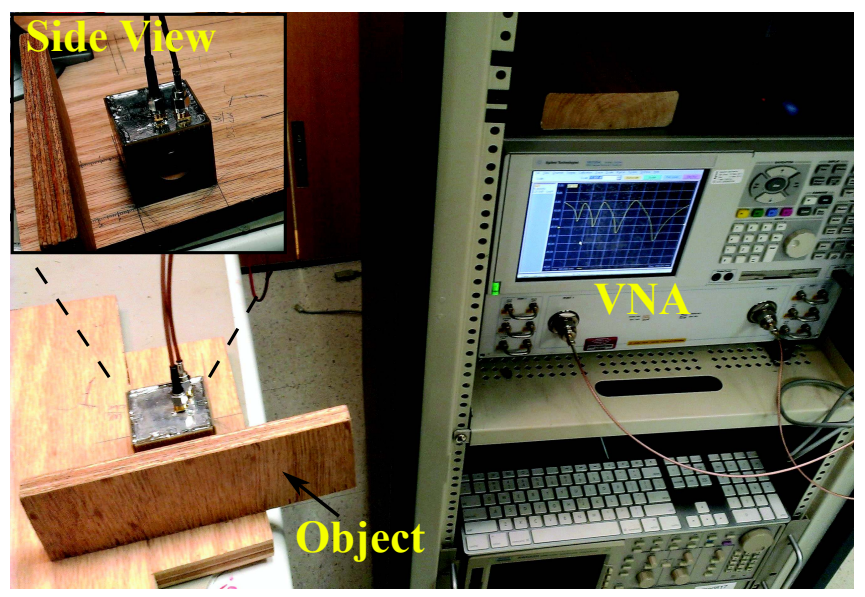

Figure 6. The measurement setup. 


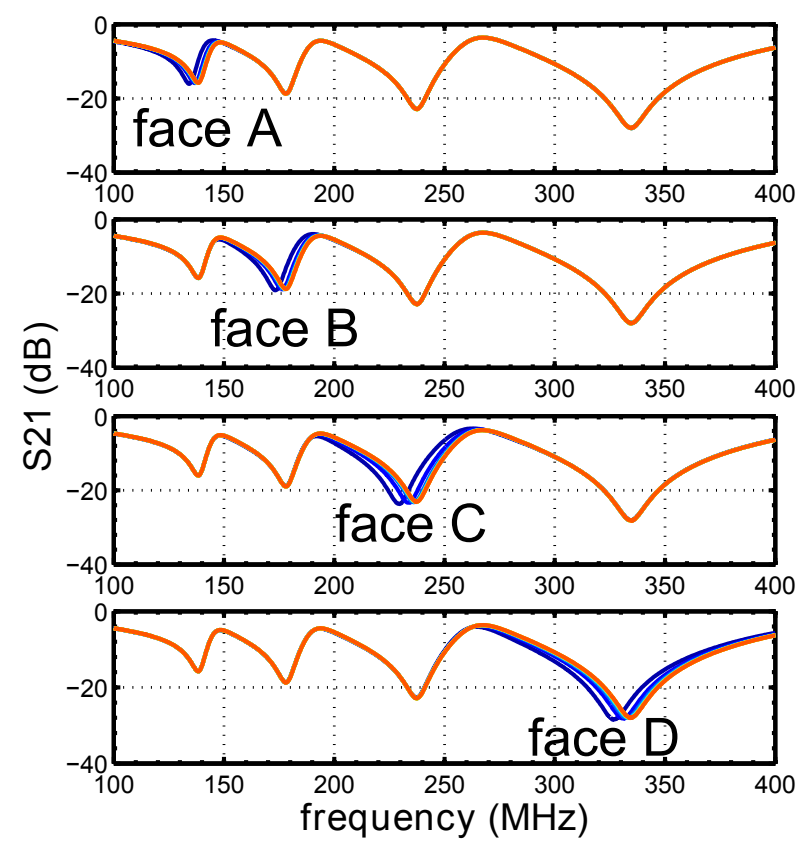

Figure 7. Transmission spectrum given by measurement results. The four sub-figures indicate the change in capacitance at four sensing elements with different inductance values: face A $\left(L_{1}=82 \mathrm{nH}\right), \mathrm{B}\left(L_{2}=56 \mathrm{nH}\right), \mathrm{C}\left(L_{3}=27 \mathrm{nH}\right), \mathrm{D}$ $\left(L_{4}=8.2 \mathrm{nH}\right)$. In each subfigure, curves with different colors represent different distances between target and sensing element.
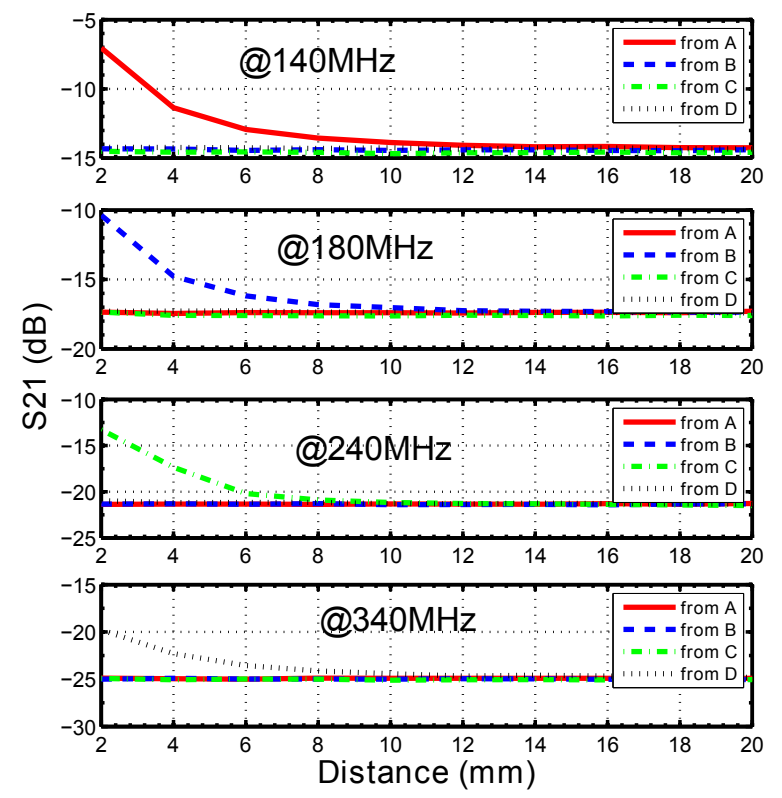

Figure 8. The measurement results. Four subfigures are for the $S_{21}$ at different frequencies. Four curves in each subfigure represent the object coming from four different directions. 


\section{CONCLUSION}

In summary, a multi-directional capacitive proximity sensor array based on band-stop filter design is proposed. A four-element capacitive proximity sensor array is integrated into a quad-band band-stop filter by arranging sensor elements with in series with lumped inductors. All of the four stop bands are decoupled with each other, which is realized by choosing different inductance values. The capacitance can be measured by measuring the shift of the corresponding stop band frequency or the change of the $S_{21}$ near the corresponding resonant frequency. A multi-direction capacitive proximity sensor is designed and fabricated. The measurement results show the capability of detecting and distinguishing target objects from different directions with a sensing range of about $8 \mathrm{~mm}$. The presented frequency-domain sensing method can be extended to other capacitive sensing array arrangements.

\section{REFERENCES}

1. J.-W. Lee, D.-J. Min, J. Kim, and W. Kim, "A 600-dpi capacitive fingerprint sensor chip and image-synthesis technique," Solid-State Circuits, IEEE Journal of 34(4), pp. 469-475, 1999.

2. T. Ding, X. Chen, and Y. Huang, "Ultra-thin flexible eddy current sensor array for gap measurements," Tsinghua Science and Technology 9(6), pp. 667-671, 2004.

3. D. Xie, Z. Huang, H. Ji, and H. Li, "An online flow pattern identification system for gas-oil two-phase flow using electrical capacitance tomography," Instrumentation and Measurement, IEEE Transactions on 55(5), pp. 1833-1838, 2006.

4. M. Sergio, N. Manaresi, M. Tartagni, R. Guerrieri, and R. Canegallo, "A textile based capacitive pressure sensor," in Sensors, 2002. Proceedings of IEEE, 2, pp. 1625-1630, IEEE, 2002.

5. P. Dietz and D. Leigh, "Diamondtouch: a multi-user touch technology," in Proceedings of the 14th annual ACM symposium on User interface software and technology, pp. 219-226, ACM, 2001.

6. R. A. Boie, L. W. Ruedisueli, and E. R. Wagner, "Computer mouse or keyboard input device utilizing capacitive sensors," Oct. 31 1995. US Patent 5,463,388.

7. R. J. Miller, S. Bisset, T. P. Allen, and G. Steinbach, "Object position and proximity detector," Feb. 27 1996. US Patent 5,495,077.

8. H. Philipp, "Capacitive sensor and array," Sept. 17 2002. US Patent 6,452,514.

9. W. Yang and A. K. Hennessey, "Security scanners with capacitance and magnetic sensor arrays," Nov. 13 2007. US Patent 7,295,019.

10. M. Oehler, V. Ling, K. Melhorn, and M. Schilling, "A multichannel portable ecg system with capacitive sensors," Physiological measurement 29(7), p. 783, 2008.

11. M. Nilsson, "Tactile sensors and other distributed sensors with minimal wiring complexity," Mechatronics, IEEE/ASME Transactions on 5(3), pp. 253-257, 2000.

12. M. Schussler, M. Puentes, C. Mandel, and R. Jakoby, "Multi-resonant perturbation method for capacitive sensing with composite right/left-handed transmission lines," in Microwave Symposium Digest (MTT), 2010 IEEE MTT-S International, pp. 481-484, IEEE, 2010.

13. Y. Yamada, K. Shin, N. Tsuchida, and M. Komai, "A tactile sensor system for universal joint sections of manipulators," Robotics and Automation, IEEE Transactions on 9(4), pp. 512-517, 1993.

14. D. M. Pepper and D. Sievenpiper, "Compact sensor using microcavity structures," Aug. 17 2004. US Patent $6,777,244$.

15. T. Lanting, H.-M. Cho, J. Clarke, M. A. Dobbs, W. Holzapfel, A. T. Lee, M. Lueker, P. Richards, A. Smith, and H. G. Spieler, "Frequency-domain squid multiplexing of transition-edge sensors," Applied Superconductivity, IEEE Transactions on 15(2), pp. 567-570, 2005.

16. W. Withayachumnankul, K. Jaruwongrungsee, C. Fumeaux, and D. Abbott, "Metamaterial-inspired multichannel thin-film sensor," Sensors Journal, IEEE 12(5), pp. 1455-1458, 2012.

17. M. Puentes, C. Weiss, M. Schussler, and R. Jakoby, "Sensor array based on split ring resonators for analysis of organic tissues," in Microwave Symposium Digest (MTT), 2011 IEEE MTT-S International, pp. 1-4, IEEE, 2011. 
18. M. Puentes, M. Maasch, M. Schussler, and R. Jakoby, "Frequency multiplexed 2-dimensional sensor array based on split-ring resonators for organic tissue analysis," Microwave Theory and Techniques, IEEE Transactions on 60(6), pp. 1720-1727, 2012.

19. X.-H. Wang, B.-Z. Wang, H. Zhang, and K. J. Chen, "A tunable bandstop resonator based on a compact slotted ground structure," Microwave Theory and Techniques, IEEE Transactions on 55(9), pp. 1912-1918, 2007.

20. J. Long, C. Li, W. Cui, J. Huangfu, and L. Ran, "A tunable microstrip bandpass filter with two independently adjustable transmission zeros," Microwave and Wireless Components Letters, IEEE 21(2), pp. 74-76, 2011.

21. Z. Wu, Y. Shim, and M. Rais-Zadeh, "Miniaturized uwb filters integrated with tunable notch filters using a silicon-based integrated passive device technology," Microwave Theory and Techniques, IEEE Transactions on $\mathbf{6 0}(3)$, pp. 518-527, 2012. 